\title{
Spin Polaron-Bipolaron Scenario of Three Gap-Like Energy Scales in Superconducting Cuprates
}

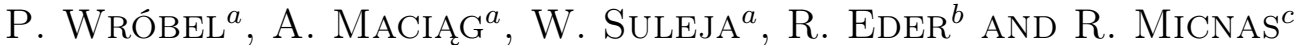 \\ ${ }^{a}$ Institute for Low Temperature and Structure Research, P.O. Box 1410, 50-950 Wrocław 2, Poland \\ ${ }^{b}$ Forschungszentrum Karlsruhe, Institut für Festkörphysik, P.O. Box 3640, D-76021 Karlsruhe, Germany \\ ${ }^{c}$ Faculty of Physics, Adam Mickiewicz University, Umultowska 85, 61-614 Poznań, Poland
}

\begin{abstract}
We argue that three gaps observed in underdoped cuprates can be attributed to the formation of antiferromagnetic spin polarons and bipolarons. Within the spin polaron scenario the antinodal pseudogap at he high energy scale originates from the change of the Fermi surface topology, induced by antiferromagnetic correlations. That change gives rise to the diminishing of the spectral weight at the antinodal region near the Brillouin zone boundary. We demonstrate that effect by analyzing effective models of doped antiferromagnets. The second type of pseudogap appearing at the intermediate energy scale originates from the phenomena which are precursory to superconductivity and predominantly concern the portion of the Fermi surface near the nodal region. In order to analyze the latter phenomenon we use the negative $U$ Hubbard model, in which many details typical to spin polaron physics are neglected, but which contains the essential ingredient of it, that is the strong short range attraction. The lowest energy scale is related to the true superconducting gap which develops with doping, although both types of pseudogap diminish with doping. This behavior can be explained by the fact that the spin polaron band is empty in the undoped system and therefore the formation of the superconducting state in the system is forbidden. Due to a pedagogical character of this report, we present in the introduction a short overview of mostly recent experimental results which are related to the gap-pseudogap physics.
\end{abstract}

PACS numbers: 74.20.Mn, 74.72.Kf

\section{Introduction}

Many data sets obtained by means of spectroscopic and transport measurements indicate that high $T_{\mathrm{c}}$ superconductors are extremely unconventional systems in comparison to standard low temperature superconductors. The nonstandard behavior manifests itself both in the normal and in the superconducting state of those materials. It seems that many aspects of that unconventional physics can be attributed to the formation of gaps in spectral densities.

In undoped systems, the spectral weight measured by means of angle resolved photoemission spectroscopy (ARPES) is lacking in the antinodal region $[1,2]$ which may be attributed to the change of the Fermi surface topology induced by antiferromagnetic correlations. In the next paragraph we will argue that this kind of physics may also influence the behavior of strongly underdoped cuprates. The tendency towards charge and spin modulations in the form of stripes may modify the spectra [3] which look in the presence of stripe order more conventional and do not change so much with the doping reduction. This seems to be the case of lanthanum based cuprates [4].

In underdoped systems, in the phase diagram region above $T_{\mathrm{c}}$, but at temperatures low enough, a second type of gap-like features has been identified from the results of various measurements. The existence of those features can be naturally interpreted in terms of uncondensed preformed pairs. The term pseudogap has been coined to describe this kind of physics. The presence of pseudogap has been established through the measurements of the nuclear magnetic resonance (NMR) [5], the scanning tunneling spectroscopy [6-9], the analysis of the electronic Raman scattering [10-12], through time-resolved optical spectroscopy [13-18], and through the ARPES [19-25]. The observed strong Nernst effect in cuprates has been also attributed to it [26-28]. Early measurements of the ${ }^{89} \mathrm{Y}$ nuclear magnetic resonance (Knight) shift in yttrium based cuprates demonstrated the existence of spin gap above $T_{\mathrm{C}}[5]$. The temperature at which this effect was observed grew with underdoping. The scanning tunneling spectroscopy allowed directly visualize the existence of a pseudogap in the high-temperature density of states measured for bismuth based compounds and other materials [29]. As the temperature is raised from $T<T_{\mathrm{c}}$ the superconducting gap remains constant and smoothly evolves into a pseudogap across $T_{\mathrm{c}}$. Coherence peaks are abruptly reduced at $T_{\mathrm{c}}$, giving rise to a generic pseudogap spectrum. Above $T_{\mathrm{c}}$, the pseudogap is gradually filling up and remains essentially constant, with a tendency to increase at higher temperature before vanishing at a crossover temperature known as $T^{*}$. Also some ARPES 
measuremets seem to provide an evidence for the direct relation between the pseudogap and pairing [25]. In the underdoped compounds, instead of a complete Fermi surface above $T_{\mathrm{c}}$, only disconnected Fermi arcs appear, separated by regions which still exhibit an energy gap. It has been suggested that in the pseudogap phase, the energy-momentum dispersion of electronic excitations near the Fermi energy behaves like the dispersion of a normal metal on the Fermi arcs and like that of a superconductor in the gaped regions.

A different set of experimental results seem to indicate that the pseudogap physics is due to some competing order. For example, recent results of electron Raman scattering indicate that these two gap-like energy scales observed in underdoped cuprates are associated with quite different excitation (quasiparticle) dynamics [30], which means that they are of different origin. Similar conclusions have been drawn from infrared ellipsometry measurements of the $c$-axis conductivity of underdoped $\mathrm{RBa}_{2} \mathrm{Cu}_{3} \mathrm{O}_{7-\delta}(\mathrm{R}=\mathrm{Y}, \mathrm{Nd}$, and La) single crystals [31]. A separate study of the in-plane magnetic penetration depth $\lambda_{a b}$ in optimally doped $(\mathrm{BiPb})_{2}(\mathrm{SrLa})_{2} \mathrm{CuO}_{6+\delta}$ which was performed by means of muon-spin rotation $(\mu \mathrm{SR})$ gives rise to similar conclusions [32]. The measurement results are inconsistent with a simple model of a $d$-wave order parameter and a uniform quasiparticle weight around the Fermi surface. It is demonstrated that the data are well described by assuming the angular gap symmetry obtained in earlier ARPES experiments [33], which suggest that the superconducting gap in the optimally doped $(\mathrm{BiPb})_{2}(\mathrm{SrLa})_{2} \mathrm{CuO}_{6+\delta}$ exists only in segments of the Fermi surface near the nodes. Thus, it is concluded that remaining parts of the Fermi surface, which are strongly affected by the pseudogap state, do not contribute significantly to the superconducting condensate. Moreover, the polarized neutron diffraction has been recently used to demonstrate that for the model superconductor $\mathrm{HgBa}_{2} \mathrm{CuO}_{4+\delta}$, a characteristic temperature marks the onset of an unusual magnetic order [34]. These results are consistent with a variant of previously proposed current-loop order and of circulating currents which induce orbital moments. Also the polarized beam neutron-scattering measurements on a highly perfect crystal of $\mathrm{YBa}_{2} \mathrm{Cu}_{3} \mathrm{O}_{6.6}$ have shown a distinct magnetic transition to a different phase with an onset at about $235 \mathrm{~K}$, the temperature expected for the pseudogap transition [35]. These data support the scenario in which the superconducting and pseudogap state are two distinct and competing phenomena. Furthermore, through a combined scanning tunneling microscopy and ARPES study, the observation of two distinct gaps (a small and a large gap) has been reported. Those gaps coexist both in real space and in the antinodal region of momentum space, below the superconducting transition temperature of $\mathrm{Bi}_{2} \mathrm{Sr}_{2-\mathrm{x}} \mathrm{La}_{\mathrm{x}} \mathrm{CuO}_{6+\delta}$ [36]. It has been shown that the small gap is associated with superconductivity. The large gap persists above $T_{\mathrm{c}}$, and seems to be linked to the observed charge ordering. Moreover, a strong correlation between the large and small gaps has been found suggesting that they are affected by similar physical processes. On the other hand, some recent ARPES measurements point out at a different origin of the pseudogap and the superconducting gap [37]. The doping and temperature dependences of the pseudogap and superconducting gap have been investigated in the single-layer cuprate $\mathrm{La}_{2-x} \mathrm{Sr}_{x} \mathrm{CuO}_{4}$ by that technique. The results clearly exhibit two distinct energy and temperature scales, namely, the gap around $(\pi, 0)$ of large magnitude and the different gap around the node characterized by the $d$-wave order parameter. In comparison with bismuth compounds having higher $T_{\mathrm{c}}$ 's, the nodal gap is smaller, while the antinodal gap and the temperature when it is formed are similar. This result suggests that the antinodal gap and the crossover temperature are approximately material-independent properties of a single $\mathrm{CuO}_{2}$ plane, in contrast to the material-dependent nodal gap, representing the pairing strength.

Contrary to the scenario of circulating currents, the results of recent $\mu \mathrm{SR}$ measurements [38] seem to indicate that dilute impurity phases are the source of the unusual magnetic orders at least in the case of $\mathrm{YBa}_{2} \mathrm{Cu}_{3} \mathrm{O}_{y}$. Furthermore, the lack of evidence for orbital-current effects in the results of the ${ }^{89} \mathrm{Y}$ NMR measurement made for the high-temperature $\mathrm{Y}_{2} \mathrm{Ba}_{4} \mathrm{Cu}_{7} \mathrm{O}_{15-\delta}$ superconductor has been recently reported [39]. Those findings do not exclude the generation of such phases in cuprates in general, but suggest that they do not have a universal character.

Summarizing this short overview of experiments regarding the pseudogap physics and their interpretations, it is tempting to notice that they provide contradicting messages. It seems that some of those contradictions can be attributed to the tendency to derive a simple physical picture and to provide arguments denying alternative scenarios suggested by opponents. In our opinion, due to complexity of cuprates in some cases seemingly contradicting scenarios might be merged into one. The examples are the pictures of preformed pairs and of circulating currents. As we have shown on the basis of the $t-J$ model the formation of bound two hole states (preformed pairs) is accompanied by the existence of current-current correlation functions forming the pattern of circulating currents [40]. This suggests that the seemingly competing phases may be just the manifestation of pair formation. On the other hand, not all the properties observed in cuprates are universal for all classes of these materials, which increases the difficulty in finding a mechanism driving the superconductivity.

In our opinion, there are three basic phenomena which give rise to gap-pseudogap physics: (i) AF correlations which induce the change of the Fermi surface topology and the depletion of charge carrier density, (ii) formation of uncondensed bound two-hole states in an effectively weakly filled band in the presence of short range attractive interaction which originates with spin fluctuations, and (iii) the true superconducting gap. In the follow- 
ing sections we will outline some elements of the theories underlying those three complementary mechanisms.

\section{Origin of the large antinodal pseudogap}

In this section we demonstrate that even in the presence of only short range antiferromagnetic correlations the Fermi surface takes the form of four oval-like shapes existing at the borders of hole pockets. Due to momentum dependence of the spectral density only four centrally located arcs are visible. In a previous work we have demonstrated a similar effect in the presence of long range antiferromagnetic order [41]. We have presented there a theory for spin-polaron-like quasiparticles. The basic idea behind this calculation was that a hole in an antiferromagnetically ordered spin background is self-trapped. This leads to a hierarchy of localized states which may also realize different irreducible representations of $C_{4 v}$. Since the Heisenberg exchange and the hopping terms have matrix elements between such self-trapped states on neighboring sites, a linear combination of atomic orbitals (LCAO) like description emerges where the role of the atomic or Wannier functions is played by the levels of the self-trapped hole. This leads to a multiband structure for the doped holes with the lowest of those being the familiar quasiparticle band observed in the insulating compounds and discussed extensively in the literature. In the mentioned work we took the point of view that the simplest description for the underdoped compounds is holes being filled into this quasiparticle band. The fact that these self-trapped states extend over several unit cells in real space necessarily implies that they have an ARPES form factor which varies within the first Brillouin zone. In addition, the strong variation of the photoemission intensity of the quasiparticle band as a function of $\boldsymbol{k}$ is induced, which explains the remnant Fermi surface and the Fermi arcs seen in ARPES. Moreover, the large antinodal pseudogap becomes a triviality within this picture. One of the higher bands of the effective LCAO Hamiltonian may have been observed in ARPES in the insulator $\mathrm{Ca}_{2} \mathrm{CuO}_{2} \mathrm{Cl}_{2}$ and optical transitions between the resulting bands may explain the mid-infrared band in optical spectroscopy. There are probably complications due to lattice polaron effects but these have been neglected although the simplicity of the calculations, which never need matrices bigger than $10 \times 10$, would certainly allow one to treat such effects as well.

Now, we will demonstrate that similar effects can be present in systems which are paramagnetic but in which short range AF correlations exist. In order to tackle the issue of the Fermi surface topology under such circumstances, in this part of the paper, we analyze the Hubbard model within an approach based on the decomposition of the square lattice into AF sublattices in which the magnetization vanishes. The approach is based on the introduction of an overcomplete set of physically relevant operators, at the price of an additional constraint which should be obeyed by them. We treat the Hamiltonian and the constraint represented in terms of those operators within the linear approximation. We also postulate that at the mean field level the sublattice magnetization vanishes in accordance with the aim to make considered scheme to be applicable to systems with short range AF correlations. We start with the definition of the representation for the physical space in terms of some new operators. If $i \in A$, where $i$ and $A$ refer to a site and to one of sublattices respectively, we postulate the following correspondence: $|0\rangle \rightarrow h_{i}^{\dagger}|0\rangle, c_{i, \uparrow}^{\dagger}|0\rangle \rightarrow|0\rangle, c_{i, \downarrow}^{\dagger}|0\rangle \rightarrow b_{i}^{\dagger}|0\rangle$, $c_{i, \downarrow}^{\dagger} c_{i, \uparrow}^{\dagger}|0\rangle \rightarrow d_{i}^{\dagger}|0\rangle$. If $j \in B$ where $j$ and $B$ refer to a site and to the second sublattice respectively, we have $|0\rangle \rightarrow h_{j}^{\dagger}|0\rangle, c_{j, \uparrow}^{\dagger}|0\rangle \rightarrow b_{j}^{\dagger}|0\rangle, c_{j, \uparrow}^{\dagger} c_{j, \downarrow}^{\dagger}|0\rangle \rightarrow d_{j}^{\dagger}|0\rangle$. The physical subspace, from which unphysical states have been removed, can be defined by the following constraint:

$$
h_{m}^{\dagger} h_{m}+b_{m}^{\dagger} b_{m}+d_{m}^{\dagger} d_{m}=0,1,
$$

or

$$
\begin{aligned}
& h_{m}^{\dagger} h_{m}+d_{m}^{\dagger} d_{m}+b_{m}^{\dagger} b_{m} \\
& \quad-\left(h_{m}^{\dagger} h_{m}+d_{m}^{\dagger} d_{m}+b_{m}^{\dagger} b_{m}\right)^{2}=0,
\end{aligned}
$$

which should be obeyed for arbitrary $m$. Since the left-hand side of (2) can be represented in terms of quartic terms only, the constraint limiting the Hilbert space reads

$$
\begin{aligned}
& -2 h_{m}^{\dagger} h_{m} d_{m}^{\dagger} d_{m}-2 h_{m}^{\dagger} h_{m} b_{m}^{\dagger} b_{m} \\
& -2 d_{m}^{\dagger} d_{m} b_{m}^{\dagger} b_{m}-b_{m}^{\dagger} b_{m}^{\dagger} b_{m} b_{m}=0,
\end{aligned}
$$

and can be neglected if we confine the analysis to the linear approximation.

The following representation of electron operators is exact inside the physical space. If $i \in A$ :

$$
\begin{aligned}
& c_{i, \uparrow}^{\dagger}=h_{i}\left(1-\hat{n}_{d, i}\right)\left(1-\hat{n}_{b, i}\right)-d_{i}^{\dagger} b_{i}, \\
& c_{i, \downarrow}^{\dagger}=h_{i} b_{i}^{\dagger}+d_{i}^{\dagger}\left(1-\hat{n}_{h, i}\right)\left(1-\hat{n}_{b, i}\right),
\end{aligned}
$$

where $\hat{n}_{d, m}=d_{m}^{\dagger} d_{m}$ etc. For $j \in B$ we obtain the symmetric representation which allows us to represent the Hamiltonian in a way that does not discriminate between sublattices

$$
\begin{gathered}
c_{j, \uparrow}^{\dagger}=h_{j} b_{j}^{\dagger}+d_{j}^{\dagger}\left(1-\hat{n}_{h, j}\right)\left(1-\hat{n}_{b, j}\right), \\
c_{j, \downarrow}^{\dagger}=h_{j}\left(1-\hat{n}_{d, j}\right)\left(1-\hat{n}_{b, j}\right)-d_{j}^{\dagger} b_{j} .
\end{gathered}
$$

It is clear that, within the linear approximation applied to hopping terms of the Hubbard Hamiltonian, only terms containing single operators representing hole or double occupancy creation or annihilation will be relevant in the last four formulae.

Quite generally we can write for the electron number operator at a given site, $m$ :

$$
c_{m, \uparrow}^{\dagger} c_{m, \uparrow}+c_{m, \downarrow}^{\dagger} c_{m, \downarrow}=1-\hat{n}_{h, m}+\hat{n}_{d, m} .
$$

Furthermore, we obtain for the magnetization operator at sites $i$ belonging to the sublattice $A$ :

$$
\hat{m}_{i, z}=\frac{1}{2}-\frac{1}{2} \hat{n}_{h, i}-\frac{1}{2} \hat{n}_{d, i}-\hat{n}_{b, i}
$$

and for the magnetization operator at sites $j$ belonging to the sublattice $B$ : 


$$
\hat{m}_{j, z}=-\frac{1}{2}+\frac{1}{2} \hat{n}_{h, j}+\frac{1}{2} \hat{n}_{d, j}+\hat{n}_{b, j} .
$$

By using the above statements we deduce that within the linear approximation the Hamiltonian including a term multiplied by a global Lagrange multiplier $\lambda$ and related to the condition of vanishing sublattice magnetization reads

$$
\begin{aligned}
& H=-t \sum_{l, \delta= \pm \hat{\boldsymbol{x}}, \pm \hat{\boldsymbol{y}}} h_{l+\delta} d_{l} \\
& -t^{\prime} \sum_{l, \delta= \pm \hat{\boldsymbol{x}} \pm \hat{\boldsymbol{y}}, \pm \hat{\boldsymbol{x}} \mp \hat{\boldsymbol{y}}}\left(h_{l+\delta} h_{l}^{\dagger}+d_{l+\delta}^{\dagger} d_{l}\right) \\
& -t^{\prime \prime} \sum_{l, \delta= \pm 2 \hat{\boldsymbol{x}}, \pm 2 \hat{\boldsymbol{y}}}\left(h_{l+\delta} h_{l}^{\dagger}+d_{l+\delta}^{\dagger} d_{l}\right) \\
& -\mu \sum_{l}\left(h_{l} h_{l}^{\dagger}+d_{l}^{\dagger} d_{l}\right)+U \sum_{l} d_{l}^{\dagger} d_{l} \\
& +\lambda \sum_{l}\left(\frac{1}{2} h_{l} h_{l}^{\dagger}-\frac{1}{2} d_{l}^{\dagger} d_{l}-b_{l}^{\dagger} b_{l}\right) .
\end{aligned}
$$

Self-consistent conditions which determine parameters $\lambda$ and $\mu$ for a given temperature $T$ and electron density $n=1-\delta$, where $\delta$ is hole filling, are

$$
\begin{aligned}
& \left\langle\sum_{l}\left(\frac{1}{2} h_{l} h_{l}^{\dagger}-\frac{1}{2} d_{l}^{\dagger} d_{l}-b_{l}^{\dagger} b_{l}\right)\right\rangle=0, \\
& \frac{\left\langle\sum_{l}\left(h_{l} h_{l}^{\dagger}+d_{l}^{\dagger} d_{l}\right)\right\rangle}{N}=n .
\end{aligned}
$$

The first condition refers to the vanishing of the sublattice magnetization in the paramagnetic state while $\langle\ldots\rangle$ denotes the average over the macrocanonical ensemble. After introducing a two-component operator $\boldsymbol{\Psi}_{m}=\left(h_{m}^{\dagger}, d_{m}\right)$ which allows us to simplify the notation we obtain a Hamiltonian which can be easily diagonalized. In order to evaluate the Green function and the spectral function within the linear approximation we use the following simplified representation for the operators creating electrons:

$$
\begin{aligned}
& i \in A: c_{i, \uparrow}=h_{i}^{\dagger}, \\
& j \in B: c_{j, \uparrow}=d_{j}, \\
& i \in A: c_{i, \downarrow}=d_{i}, \\
& j \in B: c_{j, \downarrow}=h_{j}^{\dagger} .
\end{aligned}
$$

The rest of calculation details will be presented elsewhere. In the numerical evaluation of the derived formulae we have used the following parameters: $t=0.35 \mathrm{eV}$, $t^{\prime}=0.12 \mathrm{eV}, t^{\prime \prime}=0.08 \mathrm{eV}$ and $U=12 t(4.2 \mathrm{eV})$, while in the plots we have applied Lorentzians with the broadening parameter 0.05t. In Fig. 1 the spectral function evaluated for the Fermi energy corresponding to the hole doping level $10 \%$ has been depicted. Bright colours represent regions with high values of spectral intensity. Such regions form four arcs at the border of hole pockets. In agreement with ARPES measurements the arcs are located in parts of the pocket edges which are nearest to the zone center. Since the calculation presented in this section concerns a system which is paramagnetic, the re- sult proves that even under such circumstances the Fermi surface visible in ARPES experiments may take the form of disconnected arcs.

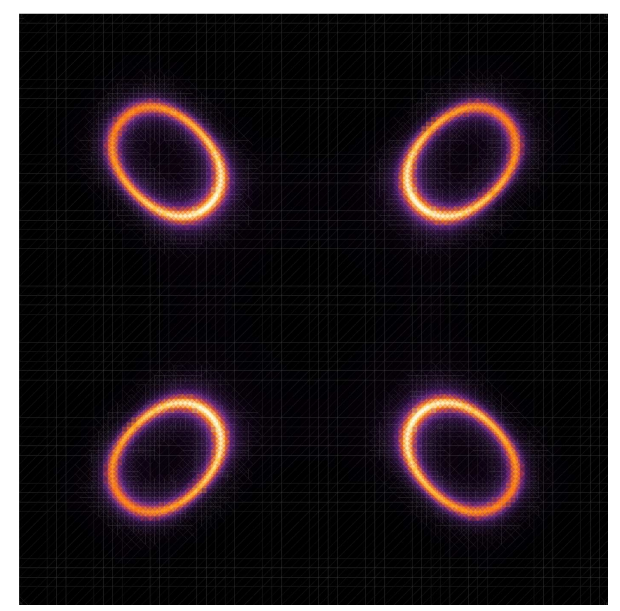

Fig. 1. Intensity plot of the spectral function at the Fermi energy in the first Brillouin zone for the doping level $10 \%$.

\section{Origin of the small nodal pseudogap}

In this section we discuss the impact which the existence of preformed pairs has on the spectral properties of the system. Within the assumed scenario, doping of an antiferromagnet is equivalent at low levels of it to filling a band formed by spin polarons. Since there exists effective attraction between particles, the formation of bound states below the two-particle continuum induces pseudogap phenomena in a weakly doped low dimensional system for which the superconducting transition is not favorable. We will demonstrate below that bound states cease to exist when the filling level increases. In our opinion this kind of behavior determines the shape of the "phase" diagram for the pseudogap physics in cuprates. There exist some additional data which confirm our scenario, and which have not been mentioned in the introduction. We will discuss them shortly here. There exists much evidence coming from the research on optical conductivity $[42,43]$ that in the low doping range the carrier density is proportional to the doped-hole concentration. The measurements of the penetration depth performed by means of the $\mu \mathrm{SR}$ technique demonstrated the proportionality between the hole density and the superfluid density [44]. This confirmed an earlier suggestion that in cuprates the scenario of the Bose-Einstein condensation in a low dimensional system with the low density of carriers may be realized $[45,46]$. Such an interpretation of $\mu \mathrm{SR}$ data is consistent with assumption that the phase transition belongs to the $n=2, X-Y$ model universality class. It means that the $T_{\mathrm{c}}$ is controlled by the phase stifness $\left(\rho_{\mathrm{S}}\right)$, not by the magnitude of the order parameter as in conventional BCS superconductors [47]. In 2D 
the proportionality between $T_{\mathrm{c}}$ and the phase stiffness is coded in the Berezinskii-Kosterlitz-Thouless (BKT) mechanism of phase transition [48], which is characterized by a universal jump of the phase stiffness.

We analyze the above described scenario for the pseudogap induced by the formation of preformed pairs in a weakly doped system in the framework of the two-dimensional negative-U Hubbard model. We draw the attention of the reader to the fact that there is no direct relation between the positive value of $U$ which we used before and the negative value od $U$ which we use now. The latter refers to some effective attraction between spin polarons, while the former to the Coulomb energy. Also the particle number $\mathrm{n}$ in negative-U Hubbard model refers now to to the density of spin polarons which is the same as hole number $\delta$. The usage of similar nomenclature has historical grounds. We treat this model as a simple version of the lattice model with short range attraction. These two features, namely low filling and short range effective attraction are most characteristic for the above discussed spin polaron (SP) model of quasiparticles and their interaction in weakly doped cuprates $[49,50]$. On the other hand, the band structure and the symmetry of possible paired states are different for negative- $U$ Hubbard and SP models. Nevertheless, in the first attempt we analyze the consequences of our pseudogap scenario within a simplest model, and neglect some details characteristic for cuprates, because the scheme suggested by us seems to be more general. To be specific, we consider the model on the square lattice, restrict the analysis to the normal state and apply the $T$-matrix approach within a simplest approximation $[51,52]$, according to which the pair susceptibility $\chi^{(0)}\left(\boldsymbol{q}, \mathrm{i} \nu_{n}\right)$ is given by

$$
\begin{aligned}
& \chi^{(0)}\left(\boldsymbol{q}, \mathrm{i} \nu_{n}\right) \\
& =\frac{1}{\beta N} \sum_{\boldsymbol{p}, \omega_{l}} \mathcal{G}^{(0)}\left(\boldsymbol{p}, \mathrm{i} \omega_{l}\right) \mathcal{G}^{(0)}\left(\boldsymbol{q}-\boldsymbol{p}, \mathrm{i} \nu_{n}-\mathrm{i} \omega_{l}\right),
\end{aligned}
$$

where $G^{(0)}\left(\boldsymbol{p}, \mathrm{i} \omega_{l}\right)$ represents the non-interacting (bare) Green function. The formula (18) is related to the representation of the $T$-matrix within the ladder approximation in terms of the bare Green functions,

$$
T\left(\boldsymbol{q}, \mathrm{i} \nu_{n}\right)=\frac{-U}{1+U \chi^{(0)}\left(\boldsymbol{q}, \mathrm{i} \nu_{n}\right)} .
$$

The next approximation which we apply is to consider only isolated poles of $T\left(\boldsymbol{q}, \mathrm{i} \nu_{n}\right)$ [53]:

$$
T\left(\boldsymbol{q}, T, \mu, \mathrm{i} \nu_{n}\right) \approx \sum_{m} \frac{R^{(m)}(\boldsymbol{q}, T, \mu)}{\mathrm{i} \nu_{n}-E_{b}^{(m)}(\boldsymbol{q}, T, \mu)} .
$$

$E_{b}^{(m)}(\boldsymbol{q}, T, \mu)$ are solutions of the equation

$$
1+U \chi^{(0)}\left(\boldsymbol{q}, E_{b}^{(m)}(\boldsymbol{q}, T, \mu)\right)=0,
$$

which for $T=0$ and just two particles determines the pair energy. The summation over the label $m$ in (20) is restricted to bound states, energies of which lie below the continuum of energies of two free particles,

$$
E_{b}^{(m)}(\boldsymbol{q}, T, \mu)+2 \mu<\min _{\boldsymbol{p} \in 1 \mathrm{BZ}}\left(t_{\boldsymbol{p}}+t_{\boldsymbol{q}-\boldsymbol{p}}\right),
$$

where $t_{\boldsymbol{p}}$ is the single-particle energy dispersion. The mean field critical temperature, which we will also evaluate in order to sketch it on the diagram for the strength of pseudogap effects can be found by solving another equation,

$$
1+U \chi^{(0)}(0,0)=0 .
$$

Following an earlier work [53] we use the number of doubly occupied sites $n_{d}$ as the measure of the pseudogap strength. Within the isolated pole approximation (20) it is given by

$$
n_{d}=-\frac{1}{N} \sum_{\boldsymbol{q}} \frac{R(\boldsymbol{q}, T, \mu)}{U^{2}} n_{\mathrm{B}}\left(E_{b}(\boldsymbol{q}, T, \mu)\right),
$$

where $n_{\mathrm{B}}$ is the Bose-Einstein distribution. In Fig. 2 a schematic phase diagram of pseudogap phenomena has been depicted. The ratio $2 n_{d} / n$, where $n$ represents the density of particles has been chosen as the measure of the pseudogap strength. By definition, the lower and upper limits of this parameter are given by 0 and 1 , respectively. It is visible at a first glance that the shape of the area in which according to our analysis pseudogap phenomena are strong, resembles the shape of the region on the phase diagram of cuprates in which the Nernst effect is strong [26-28].

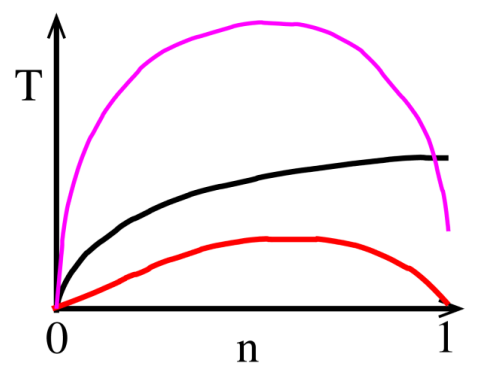

Fig. 2. Schematic diagram for pseudogap phenomena induced upon doping the system with strong short range attraction. The dome bordered by the upper curve represents the region when the ratio $2 n_{d} / n$ substantially differs from zero. The second (from top) curve represents the BCS critical temperature derived from the Thouless criterion (23). The lowest curve schematizes the temperature of the BKT transition applicable to two-dimensional systems.

\section{Discussion}

The presented scenario based on the idea of filling the spin polaron band which is empty in undoped systems, seems at least qualitatively to explain several phenomena which belong to the category of pseudogap effects. Also the behavior of the superconducting gap which grows with doping in underdoped cuprates is in agreement with the spin-polaron scheme $[49,50]$. This effect can be attributed to the known fact that at low filling levels the superfluid density strongly depends on the density. The disappearance of the superconducting order parameter in overdoped systems should be attributed, according to 
our scenario, to the vanishing of antiferromagnet fluctuations, because the exchange of magnons by holes provides glue for pairing. For doping levels at which such vanishing takes place our approach being applicable to systems with the substantial size of the antiferromagnetic correlation length ceases to be valid. Nevertheless, our scenario is even in that doping range consistent with experimental observations showing more conventional behavior and disappearance of superconductivity. Recently, it has been announced that in multilayered heterogeneous oxide structures it is possible to induce a triplet component in a cuprate superconductor [54]. The proximity to the stabilization of a superconducting state with a $p$-wave component has been also predicted in the framework of the spin polaron approach [55].

It is clear that lattice effects influence the behavior of cuprates [56]. As it has been shown by the Zürich group, the isotope effect is seen for both $T_{\mathrm{c}}$ and the superfluid density [57]. The interplay between spin polarons - bipolarons and phonons needs to be analyzed more thoroughfully. Nevertheless, it is clear already now that the lattice effects profoundly influence the properties of these systems.

In summary, the phenomenology of gaps observed in cuprates seems to agree with the concept of spin polaron formation in doped antiferromagnets. The large antinodal gap is the manifestation of the Fermi surface topology change induced by antiferromagnetic fluctuations. Some additional complications may bring about the tendency toward charge ordering in the form of stripes or even in the form of a checkerboard [58]. Such a tendency is also seen in models based on the spin polaron approach. A more detailed analysis suggests that the so-called checkerboard structure is an averaged over directions form of one-dimensional charge modulations in which preformed pairs participate [59]. The experimentally observed tendency toward more conventional shape of the Fermi surface in systems in which stripes are formed also agrees with analyzes based on the spin polaron idea. The nodal and smaller pseudogap is attributed, within that scenario, to precursory effects to pairing. Their appearance is induced by the fact that spin polaron filling is relatively low in underdoped systems. With doping the systems become more conventional because antiferromagnetic fluctuations weaken and electron correlation effects become less important due to higher average distance between electrons and due to disappearance of the attraction mechanism for holes.

\section{References}

[1] F. Ronning, C. Kim, D.L. Feng, D.S. Marshall, A.G. Loeser, L.L. Miller, J.N. Eckstein, I. Bozovic, Z.-X. Shen, Science 282, 2067 (1998).

[2] F. Ronning, C. Kim, K.M. Shen, N.P. Armitage, A. Damascelli, D.H. Lu, D.L. Feng, Z.-X. Shen, L.L. Miller, Y.-J. Kim, F. Chou, I. Terasaki, Phys. Rev. B 67, 035113 (2003).
[3] P. Wróbel, A. Maciąg, R. Eder, J. Phys., Condens. Matter 18, 9749 (2006).

[4] A. Ino, C. Kim, M. Nakamura, T. Yoshida, T. Mizokawa, Z.-X. Shen, A. Fujimori, T. Kakeshita, H. Eisaki, S. Uchida, Phys. Rev. B 62, 4137 (2000).

[5] H. Alloul, A. Mahajan, H. Casalta, O. Klein, Phys. Rev. Lett. 70, 1171 (1993).

[6] C. Renner, B. Revaz, J.-Y. Genoud, K. Kadowaki, Ø. Fischer, Phys. Rev. Lett. 80, 149 (1998).

[7] A. Matsuda, S. Sugita, T. Watanabe, Phys. Rev. B 60, 1377 (1999).

[8] I. Maggio-Aprile, Ch. Renner, A. Erb, E. Walker, B. Revaz, J.Y. Genoud, K. Kadowaki, Ø. Fischer, J. Electron Spectrosc. Relat. Phenom. 109, 147 (2000).

[9] M. Kugler, Ø. Fischer, Ch. Renner, S. Ono, Y. Ando, Phys. Rev. Lett. 86, 4911 (2001).

[10] T. Staufer, R. Hackl, P. Müller, Solid State Commun. 75, 975 (1990)

[11] R. Hackl, M. Opel, P.F. Muller, G. Krug, B. Stadlober, R. Nemetschek, H. Berger, L. Forro, J. Low Temp. Phys. 105, 733 (1996).

[12] Y.H. Liu, K. Takeyama, T. Kurosawa, N. Momono, M. Oda, M. Ido, Phys. Rev. Lett. 101, 137003 (2008).

[13] T.N. Thomas, C.J. Stevens, A.J.S. Choudary, J.F. Ryan, D. Mihailovic, T. Mertelj, L. Forro, G. Wagner, J.E. Evetts, Phys. Rev. B 53, 12436 (1996).

[14] C.J. Stevens, D. Smith, C. Chen, J.F. Ryan, B. Podobnik, D. Mihailovic, G.A. Wagner, J.E. Evetts, Phys. Rev. Lett. 78, 2212 (1997).

[15] V.V. Kabanov, J. Demsar, B. Podobnik, D. Mihailovic, Phys. Rev. B 59, 1497 (1999).

[16] J. Demsar, B. Podobnik, V.V. Kabanov, Th. Wolf, D. Mihailovic, Phys. Rev. Lett. 82, 4918 (1999).

[17] H. Murakami, T. Kiwa, N. Kida, M. Tonouchi, T. Uchiyama, I. Iguchi, Z. Wang, Europhys. Lett. 60 , 288 (2002).

[18] N. Gedik, M. Langner, J. Orenstein, S. Ono, Y. Abe, Y. Ando, Phys. Rev. Lett. 95, 117005 (2005).

[19] D.S. Marshall, D.S. Dessau, A.G. Loeser, C.-H. Park, A.Y. Matsuura, J.N. Eckstein, I. Bozovic, P. Fournier, A. Kapitulnik, W.E. Spicer, Z.-X. Shen, Phys. Rev. Lett. 76, 4841 (1996).

[20] H. Ding, T. Yokoya, J.C. Campuzano, T. Takahashi, M. Randeria, M.R. Norman, T. Mochiku, K. Kadowaki, J. Giapintzakis, Nature (London) $\mathbf{3 8 2}$, 51 (1996).

[21] H. Ding, M.R. Norman, J.C. Campuzano, M. Randeria, A.F. Bellman, T. Yokoya, T. Takahashi, T. Mochiku, K. Kadowaki, Phys. Rev. B 54, 9678 (1996).

[22] M.R. Norman, H. Ding, M. Randeria, J.C. Campuzano, T. Yokoya, T. Takeuchi, T. Takahashi, T. Mochiku, K. Kadowaki, P. Guptasarma, D.G. Hinks, Nature 392, 157 (1998).

[23] A. Kanigel, M.R. Norman, M. Randeria, U. Chatterjee, S. Souma, A. Kaminski, H.M. Fretwell, S. Rosenkranz, M. Shi, T. Sato, T. Takahashi, Z.Z. Li, H. Raffy, K. Kadowaki, D. Hinks, L. Ozyuzer, J.C. Campuzano, Nature Phys. 2, 447 (2006). 
[24] A. Kanigel, U. Chatterjee, M. Randeria, M.R. Norman, S. Souma, M. Shi, Z.Z. Li, H. Raffy, J.C. Campuzano, Phys. Rev. Lett. 99, 157001 (2007).

[25] A. Kanigel, U. Chatterjee, M. Randeria, M.R. Norman, G. Koren, K. Kadowaki, J.C. Campuzano, Phys. Rev. Lett. 101, 137002 (2008).

[26] Yayu Wang, Lu Li, M.J. Naughton, G.D. Gu, S. Uchida, N.P. Ong, Phys. Rev. Lett. 95, 247002 (2005).

[27] Y. Wang, L. Li, N.P. Ong, Phys. Rev. B 73, 024510 (2006).

[28] L. Li, Y. Wang, S. Komiya, S. Ono, Y. Ando, G.D. Gu, N.P. Ong, Phys. Rev. B 81, 054510 (2010).

[29] Ø. Fischer, M. Kugler, I. Maggio-Aprile, C. Berthod, Rev. Mod. Phys. 79, 353 (2007).

[30] M. Le Tacon, A. Sacuto, A. Georges, G. Kotliar, Y. Gallais, D. Colson, A. Forget, Nature Phys. 2, 537 (2006).

[31] L. Yu, D. Munzar, A.V. Boris, P. Yordanov, J. Chaloupka, Th. Wolf, C.T. Lin, B. Keimer, C. Bernhard, Phys. Rev. Lett. 100, 177004 (2008).

[32] R. Khasanov, Takeshi Kondo, S. Strässle, D.O.G. Heron, A. Kaminski, H. Keller, S.L. Lee, T. Takeuchi, Phys. Rev. Lett. 101, 227002 (2008).

[33] T. Kondo, T. Takeuchi, A. Kaminski, S. Tsuda, S. Shin, Phys. Rev. Lett. 98, 267004 (2007).

[34] Y. Li, V. Baledent, N. Barisic, Y. Cho, B. Fauqué, Y. Sidis, G. Yu, X. Zhao, P. Bourges, M. Greven, Nature 455, 372 (2008).

[35] H.A. Mook, Y. Sidis, B. Fauque, V. Baledent, P. Bourges, Phys. Rev. B 78, 020506(R) (2008).

[36] J.-H. Ma, Z.-H. Pan, F.C. Niestemski, M. Neupane, Y.-M. Xu, P. Richard, K. Nakayama, T. Sato T. Takahashi, H.-Q. Luo, L. Fang, H.-H. Wen, Z. Wang, H. Ding, V. Madhavan, Phys. Rev. Lett. 101, 207002 (2008)

[37] T. Yoshida, M. Hashimoto, S. Ideta, A. Fujimori, K. Tanaka, N. Mannella, Z. Hussain, Z.-X. Shen, M. Kubota, K. Ono, S. Komiya, Y. Ando, H. Eisaki, S. Uchida, Phys. Rev. Lett. 103, 037004 (2009).

[38] J.E. Sonier, V. Pacradouni, S.A. Sabok-Sayr, W.N. Hardy, D.A. Bonn, R. Liang, H.A. Mook, Phys. Rev. Lett. 103, 167002 (2009).

[39] S. Strässle, J. Roos, M. Mali, H. Keller, Phys. Rev. Lett. 101, 237001 (2008).

[40] P. Wróbel, R. Eder, Phys. Rev. B 64, 184504 (2001).

[41] P. Wróbel, W. Suleja, R. Eder, Phys. Rev. B $\mathbf{7 8}$ 064501 (2008).

[42] J. Orenstein, G.A. Thomas, A.J. Millis, S.L. Cooper, D.H. Rapkine, T. Timusk, L.F. Schneemeyer, J.V. Waszczak, Phys. Rev. B 42, 6342 (1990).
[43] S. Uchida, T. Ido, H. Takagi, T. Arima, Y. Tokura, S. Tajima, Phys. Rev. B 43, 7942 (1991).

[44] Y.J. Uemura, G.M. Luke, B.J. Sternlieb, J.H. Brewer J.F. Carolan, W.N. Hardy, R. Kadono, J.R. Kempton, R.F. Kiefl, S.R. Kreitzman, P. Mulhern, T.M. Riseman, D.L. Williams, B.X. Yang, S. Uchida, H. Takagi, J. Gopalakrishnan, A.W. Sleight, M.A. Subramanian, C.L. Chien, M.Z. Cieplak, Gang Xiao, V.Y. Lee, B.W. Statt, C.E. Stronach, W.J. Kossler, X.H. Yu, Phys. Rev. Lett. 62, 2317 (1989).

[45] R. Micnas, J. Ranninger, S. Robaszkiewicz, S. Tabor, Phys. Rev. B 37, 9410 (1988); R. Micnas, J. Ranninger, S. Robaszkiewicz, Rev. Mod. Phys. 62, 113 (1990).

[46] R. Micnas, S. Robaszkiewicz, in: High-T $T_{\mathrm{c}}$ Superconductivity 1996: Ten Years after the Discovery, Eds. E. Kaldis, E. Liarokapis, K.A. Müller, NATO ASI Series, Kluwer Academic Publishers, Dordrecht 1997, Vol. 343 p. 31, and refs. therein.

[47] V.J. Emery, S. Kivelson, Nature 374, 434 (1995).

[48] D.J. Nelson, J. Kosterlitz, Phys. Rev. Lett. 37, 1201 (1977).

[49] P. Wróbel, R. Eder, R. Micnas, J. Phys., Condens. Matter 15, 2755 (2003).

[50] P. Wróbel, R. Eder, P. Fulde, J. Phys., Condens. Matter 15, 6599 (2003).

[51] P. Nozières, S. Schmitt-Rink, J. Low. Temp. Phys. 59, 195 (1985).

[52] R. Micnas, M.H. Pedersen, S. Schafroth, T. Schneider, J.J. Rodriguez-Nunez, H. Beck, Phys. Rev. B 52, 16223 (1995).

[53] M.Yu. Kagan, R. Frésard, M. Capezzali, H. Beck, Phys. Rev. B 57, 5995 (1998).

[54] K. Dybko, K. Werner-Malento, P. Aleshkevych, M. Wojcik, M. Sawicki, P. Przyslupski, Phys. Rev. B 80, 144504 (2009); and this volume.

[55] A. Maciąg, P. Wróbel, Phys. Status Solidi B 243, 512 (2006)

[56] A.S. Mishchenko, Adv. Condens. Matter Phys. 2010, 306106 (2010).

[57] H. Keller, A. Bussmann-Holder, Adv. Condens. Matter Phys. 2010, 393526 (2010).

[58] K. McElroy, D.-H. Lee, J.E. Hoffman, K.M. Lang, J. Lee, E.W. Hudson, H. Eisaki, S. Uchida, J.C. Davis, Phys. Rev. Lett. 94, 197005 (2005); and references therein.

[59] P. Wróbel, Phys. Rev. B 74, 014507 (2006). 